$02,05,13$

\title{
О магнитометрическом определении концентрации радиационных дефектов в сверхпроводящей пленке $\mathrm{GdBa}_{2} \mathrm{Cu}_{3} \mathrm{O}_{7-x}$
}

\author{
(С) А.И. Подливаев ${ }^{1,2}$, И.А. Руднев ${ }^{1}$ \\ ${ }^{1}$ Национальный исследовательский ядерный университет „МИФИ“, \\ Москва, Россия \\ ${ }^{2}$ Научно-исследовательский институт проблем развития научно-образовательного потенциала молодежи, \\ Москва, Россия \\ E-mail: AlPodlivayev@mephi.ru
}

Поступила в Редакцию 11 февраля 2021 г.

В окончательной редакции 11 февраля 2021 г.

Принята к публикации 17 февраля 2021 г.

Предложена и обоснована экспрессная магнитометрическая методика, позволяющая определять неоднородность изменения сверхпроводящих свойств тонкого слоя сверхпроводника, обусловленную радиационным облучением. Проведено численное моделирование этой методики на примере сверхпроводящего слоя $\mathrm{GdBa}_{2} \mathrm{Cu}_{3} \mathrm{O}_{7-x}$, облучаемого ионами водорода и гелия. Расчет профиля концентрации радиационных дефектов проводился с использованием комплекса программ SRIM. Расчет распределения плотности сверхпроводящих токов, индуцированных внешним магнитным полем проводился в рамках модели критического состояния. Модельные расчеты показали эффективность предлагаемой методики.

Ключевые слова:сверхпроводник, облучение, радиационные дефекты, магнитометрия, критический ток, $\mathrm{GdBaCuO}$.

DOI: 10.21883/FTT.2021.06.50927.028

\section{1. Введение}

Современные композитные высокотемпературные сверхпроводники (ВТСП), в частности, ВТСП-ленты второго поколения на основе соединений $R e \mathrm{Ba}_{2} \mathrm{Cu}_{3} \mathrm{O}_{7-x}$ $(R e \mathrm{BaCuO}$, где $R e-$ редкоземельный элемент) обладают высокими значениями критического тока, благодаря чему они на данный момент широко используются в первую очередь в области электроэнергетики, позволяя создавать высокоэффективные линии электропередачи, моторы и генераторы. ВТСП-композиты также обладают высокими значениями плотности критического тока в сильных магнитных полях, что делает их крайне перспективным материалом для использования в сверхпроводящих магнитных системах (CMC), различных установках класса МЕГА-сайнс (большой адронный коллайдер БАК, ускоритель НИКА, проект ИТЭР и др.). В этих установках ВТСП находятся в условиях сильных радиационных полей, неизбежно приводящих к изменению сверхпроводящих характеристик, прежде всего критического тока и критической температуры, из-за образования в ВТСП-материале радиационных дефектов.

Неоднородность распределения дефектов в сверхпроводнике может приводить к локальной неоднородности изменения сверхпроводящих характеристик в объеме материала. В свою очередь такая неоднородность может приводить к необычным физическим явлениям, например, появлению фазовых переходов по току, аналогичных наблюдаемым в гранулированных сверхпроводниках $\mathrm{YBa}_{2} \mathrm{Cu}_{3} \mathrm{O}_{7-x}$ [1]. В настоящей работе предложена и обоснована экспрессная магнитометрическая методика, позволяющая определять неоднородность изменения сверхпроводящих свойств тонкого слоя сверхпроводника $\mathrm{GdBa}_{2} \mathrm{Cu}_{3} \mathrm{O}_{7-x}$ (GdBaCuO123), вызванную радиационным воздействием.

\section{2. Постановка задачи}

В реальных СМС, работающих в радиационных полях, обусловленных термоядерным синтезом, повреждение элемента сверхпроводящей обмотки является однородным. Однако в имитационных экспериментах, проводимых на ускорителях заряженных частиц, замена потока нейтронов или $\gamma$-частиц потоком заряженных ионов со сравнительно низкой длиной пробега, однородность распределения радиационных дефектов по толщине ВТСП слоя может быть нарушена. Высокая стоимость одного часа облучения образца требует во-первых предварительной теоретической оценки режима облучения, который обеспечивает удовлетворительный уровень однородности концентрации дефектов, и, во-вторых, разработки экспериментальной методики контроля этой однородности. В представленной работе расчет концентрации радиационных дефектов проводится с привлечением программного пакета SRIM (Stopping and Range of Ions in Matter [2]). Неоднородное радиационное облучение приводит к неоднородной деградации сверхпроводящих свойств ВТСП-слоя, которая может быть определена магнитометрической методикой, в которой критические токи в сверхпроводнике наводятся внешним магнитным полем и определяются по магнитному отклику образца. 


\section{1. Характеристики образца и режим облучения}

При расчете накопления радиационных дефектов и при магнитометрических измерениях в качестве мишени рассматривался квадратный фрагмент ВТСП-ленты $\mathrm{GdBa}_{2} \mathrm{Cu}_{3} \mathrm{O}_{7-x}$ производства фирмы СуперОкс. Размер образца - $L \times L, L=4 \mathrm{~mm}$. В отсутствие внешнего магнитного поля величина объемной плотности критического тока при температуре жидкого азота $J_{c}=2.77 \cdot 10^{6} \mathrm{~A} / \mathrm{cm}^{2}$ [3]. Схематическая структура образца, направления координатных осей и потока облучающих частиц представлены на рис. 1. Декартова система $(X, Y, Z)$ выбрана так, что координаты ВТСП-слоя удовлетворяют условиям $-L / 2 \leq X, Y \leq L / 2, \leq Z \leq h$. Толщина верхнего слоя меди, слоев серебра и сверхпроводника равны, соответственно 20,2 , и $1.5 \mu \mathrm{m}$, соответственно. Эти слои расположены на подложке из сплава Hastelloy C276 (Ni, Cr, Мо и др.), которая отделена от сверхпроводника тонкой буферной прослойкой из оксидов металлов.

Для описания критических токов в тонкой сверхпроводящей пленке с неоднородными по толщине характеристиками можно использовать подход, основанный на решении уравнений Гинзбурга-Ландау [4], однако в представленной работе применяется альтернативная постановка задачи. Введем величину двумерной плотности критического тока $j_{c}\left(B_{Z}\right)$. Связь трехмерной плотности критического тока $J_{c}(Z)$ и величины $j_{c}\left(B_{Z}\right)$ определяется следующим образом:

$$
j_{c}\left(B_{Z}\right)=\int_{0}^{h} J_{c}(Z) d z,
$$

где $h$ - толщина сверхпроводящего слоя.

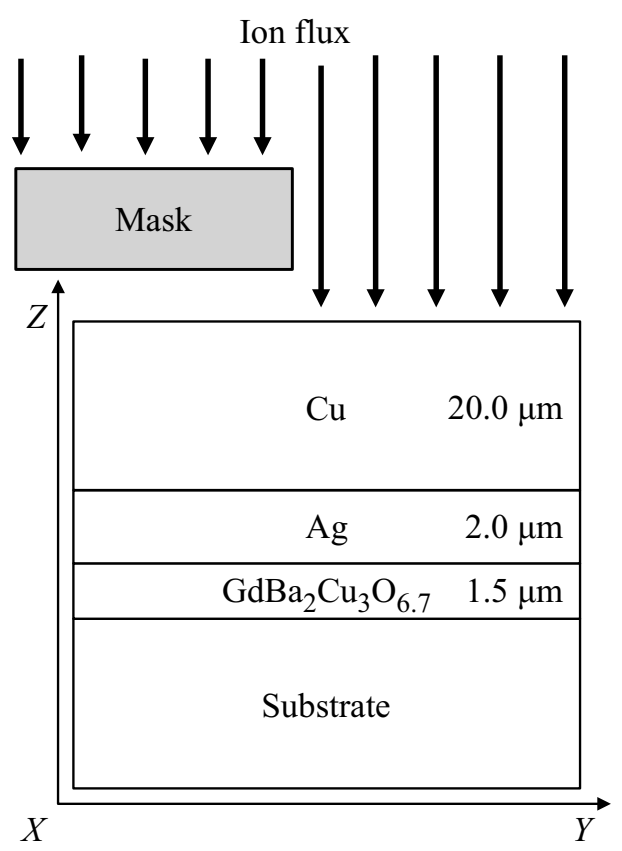

Рис. 1. Структура композитного образца.
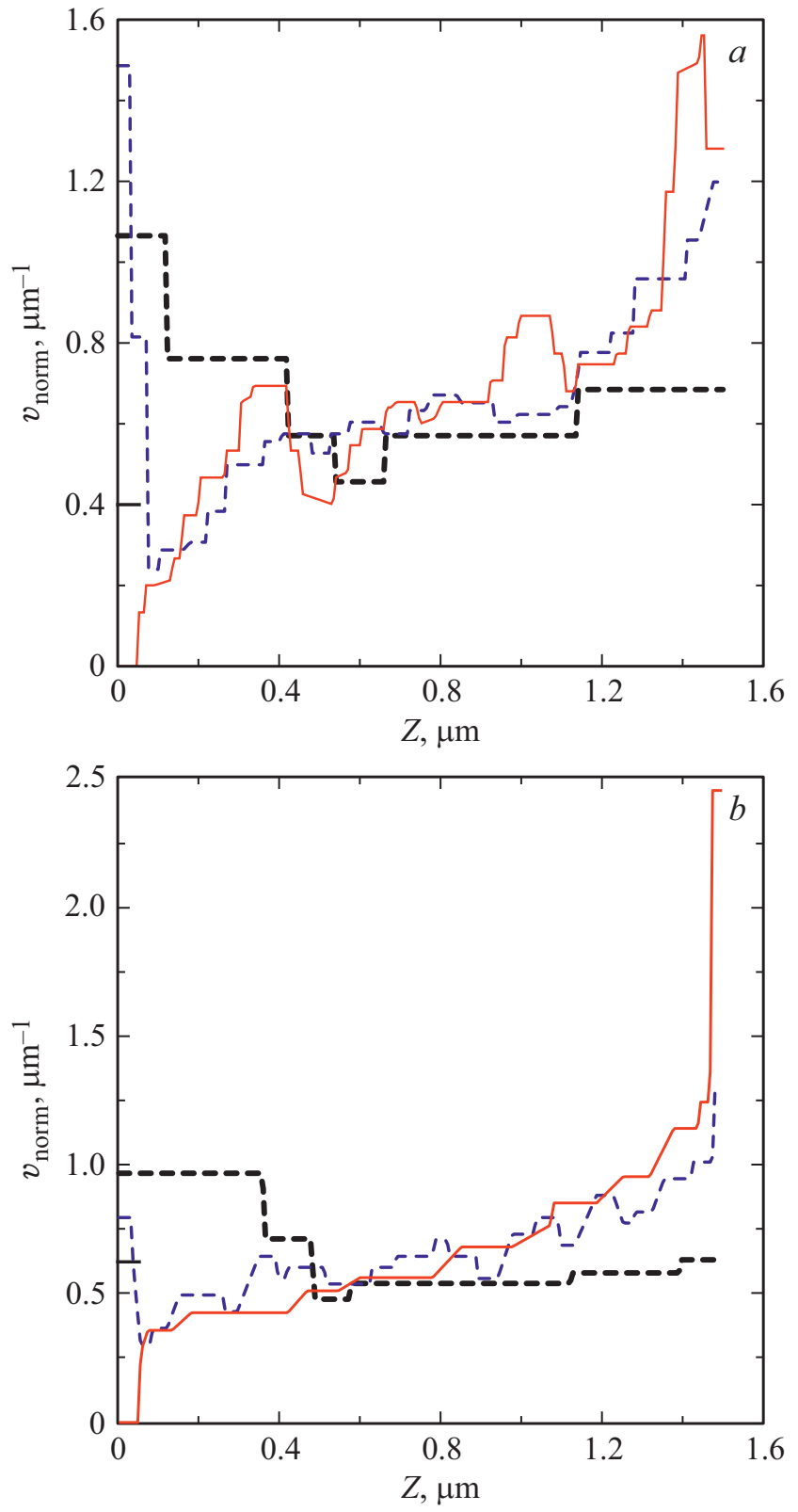

Рис. 2. Нормированная плотность радиационных вакансий при облучении ионами водорода $(a)$ и гелия $(b)$. Жирная прерывистая черная линия - образец I. Средняя прерывистая синяя линия - образец II. Тонкая сплошная красная линия образец III.

В отсутствие радиационных повреждений при величине объемной плотности $J_{c}=2.77 \cdot 10^{6} \mathrm{~A} / \mathrm{cm}^{2}$ и толщине ВТСП-слоя и $h=1.5 \mu \mathrm{m}$, поверхностная плотность критического тока $j_{c}$ образца равна $41548 \mathrm{~A} / \mathrm{m}$. Применение при облучении экранирующей маски (см. рис. 1) позволяет одновременно сопоставить характеристики исходного образца и облученной области. Это сокращает количество магнитометрических измерений, делая их результаты более достоверными.

Расчет повреждений проводился для трех типов образцов. В образце I сверхпроводящий слой закрыт слоем 
Энергии ионов $(\mathrm{keV})$

\begin{tabular}{c|c|c|c}
\hline Параметр & Образец I & Образец II & Образец III \\
\hline $\mathrm{H}$ & 2400 & 600 & 300 \\
$\mathrm{He}$ & 9500 & 2200 & 550
\end{tabular}

меди и слоем серебра (см. рис. 1). В образце II удален медный, а в образце III - и медный и серебряный слои. При облучении предполагалось использование ионов водорода и гелия. Энергии ионов для трех образцов приведены в таблице. При таких энергиях 95-98\% быстрых частиц проходят через сверхпроводящий слой и останавливаются в подложке. Расчет проводился для 100000 частиц, падающих на композит. Этого количества достаточно для того, чтобы дисперсией плотности распределения радиационных вакансий $v(Z)$ в сверхпроводящем слое можно было пренебречь. На рис. 2 представлены зависимости нормированной плотности вакансий $v_{\text {norm }}(Z)$ от вертикальной координаты $Z$, определяемой следующим образом:

$$
v_{\text {norm }}(Z)=v(z) / \int_{0}^{h} v\left(Z^{\prime}\right) d Z^{\prime}
$$

где $h=1.5 \mu \mathrm{m}$ - толщина сверхпроводящего слоя. В модельных расчетах настоящей работы предполагается следующая линейная зависимость деградации объемной плотности критического тока от концентрации радиационных дефектов

$$
J_{c}(v)= \begin{cases}J_{c}(0) \cdot\left(1-v / v_{\max }\right) ; & v \leq v_{\max } \\ 0 ; & v>v_{\max }\end{cases}
$$

где $v_{\max }-$ предельная концентрация радиационных дефектов, при которой полностью разрушается сверхпроводимость. Это приближение вполне соответствует экспериментальным данным, полученным при однородном радиационном повреждении различных ВТСП-образцов (см. работу [5] и ссылки в ней).

\section{2. Основные уравнения магнитометрической методики}

В настоящее время применяются различные модификации магнитометрических методик определения двумерной плотности критического тока пленок ВТСП (см., например, обзор [6] и ссылки в нем). Общим элементом всех методик является измерении нормальной компоненты индукции магнитного поля $B_{Z}(X, Y, Z)$ над поверхностью пленки, и восстановление из этих данных распределения плотности двумерного тока в пленке. Связь токов с магнитным полем определяется следующим уравнением Био-Савара

$$
\begin{aligned}
B_{Z}(X Y Z)= & B_{\text {ext }}+\frac{\mu_{0}}{4 \pi} \\
& \times \int_{S} \frac{j_{X}^{\left(X^{\prime}, Y^{\prime}\right)\left(Y-Y^{\prime}\right)}-j_{Y}^{\left(X^{\prime}, Y^{\prime}\right)\left(X-X^{\prime}\right)}}{\left(\left(X-X^{\prime}\right)^{2}\left(Y-Y^{\prime}\right)^{2}+Z^{2}\right)^{3 / 2}} d X^{\prime} d Y^{\prime},
\end{aligned}
$$

где $j_{X}, j_{Y}-$ являются соответствующими компонентами поверхностных токов в единицах $\mathrm{A} / \mathrm{m}$. Интегрирование ведется по поверхности ленты, $\mu_{0}$ - магнитная проницаемость вакуума, $B_{\text {ext }}-$ нормальная компонента внешнего магнитного поля, не связанного со сверхпроводящими токами пленки.

В настоящей работе нас интересует бесконтактная методика, при которой в образце отсутствует транспортный ток, а сверхпроводящие токи наводятся только внешним магнитным полем. В этом случае линии двумерных токов в пленке замкнуты, и токовые компоненты $j_{X}, j_{Y}$ могут быть выражены через плотность нормальной компоненты магнитного момента пленки $g(X, Y)$ следующим образом [6,7]:

$$
\begin{gathered}
j_{X}(X, Y, t)=\frac{\partial g(X, Y, t)}{\partial Y} ; \\
j_{Y}(X, Y, t)=-\frac{\partial g(X, Y, t)}{\partial X} .
\end{gathered}
$$

(Здесь $t$ - время). В рамках этой замены мы, получаем из (3), (4) следующую модификацию уравнения Био-Савара:

$$
\begin{gathered}
B_{Z}(X, Y, Z, t)=B_{\text {ext }}(t)+\mu_{0} \int_{S} K\left(r, r^{\prime}\right) g\left(X, X^{\prime}, t\right) d X^{\prime} d Y^{\prime}, \\
K\left(r, r^{\prime}\right)=\frac{1}{4 \pi} \frac{2 Z^{2}-\left(X-X^{\prime}\right)^{2}-\left(Y-Y^{\prime}\right)^{2}}{\left[Z^{2}+\left(X-X^{\prime}\right)^{2}+\left(Y-Y^{\prime}\right)^{2}\right]^{5 / 2}},
\end{gathered}
$$

где $K\left(r, r^{\prime}\right)$ - ядро интегрального оператора, радиусвектор.

При исследовании сверхпроводимости тонких пленок уравнение (4) в различных работах применяется для решения двух групп противоположных задач (см., например, обзор [6] и ссылки в нем).

К первой группе работ относится магнитометрическая методика определения плотности наведенного в ВТСПпленке тока по измеренному внешнему магнитному полю. В этом случае решается так называемая обратная задача Био-Савара. Обратная задача относится к классу некорректных и требует специальных методов решения $[8,9]$. Конечной целью решения этой задачи является определение плотности критического тока в пленке. В соответствие с моделью критического состояния $[10,11]$, модуль плотности наведенного тока $\sqrt{j_{X}^{2}(X, Y)+j_{Y}^{2}(X, Y)}$ не равен плотности поверхностного критического тока $j_{c}$, но при высоких амплитудах внешнего магнитного поля область, где эти величины существенно различаются пренебрежимо мала. 


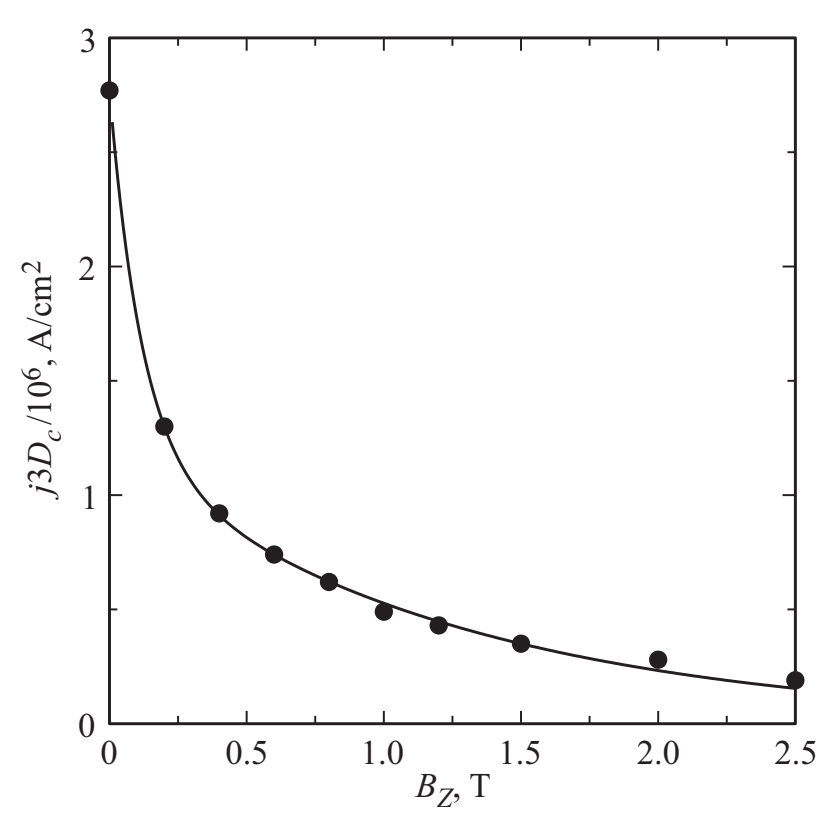

Рис. 3. Зависимость трехмерной плотности критического тока от нормальной компоненты индукции магнитного поля. Черные кружки - данные экспериментальной работы [2].

Ко второй группе относятся задачи, в которых исходно задано распределение плотности критического тока $j_{c}$ как функции координат и магнитного поля, временной закон изменения внешнего магнитного поля, а также материальные уравнения (аналог закона Ома). При решении этих задач определяется плотность наведенного магнитного момента $g(X, Y, t)$ и соответствующие токовые компоненты $j X, j Y$. Представленная в данной работе задача относится к этой группе. Зависимость трехмерной плотности критического тока $J_{c}$ от концентрации радиационных дефектов определяется выражением (3), а зависимость от величины индукции магнитного поля апроксимируется двухэкспоненциальным приближением [12]. При таком подходе выражение (3) принимает следующий вид:

$$
\begin{gathered}
J_{c}\left(v, B_{Z}\right)= \begin{cases}C\left(B_{Z}\right) \cdot\left(1-v / v_{\max }\right) / h, & v \leq v_{\max }, \\
0, & v>v_{\max }\end{cases} \\
C(B)=\left(A_{1} \cdot \exp \left(-|B| / \beta_{1}\right)+A_{2} \cdot \exp \left(-|B| / \beta_{2}\right)\right)^{\prime} .
\end{gathered}
$$

Параметры приближения $A_{1}=23665 \mathrm{~A} / \mathrm{m}, A_{2}=$ $=17884 \mathrm{~A} / \mathrm{m}, \quad \beta_{1}=0.1175 \mathrm{~T}$ и $\beta_{2}=1.2238 \mathrm{~T}$ определяются подгонкой к экспериментальной зависимости работы [3] методом наименьших квадратов. На рис. 3 представлена зависимость (6) и соответствующая ей экспериментальная зависимость, где видно хорошее совпадение экспериментальных данных и аппроксимирующей кривой. В работе [12] было показано, что двухэкспоненциальное приближение более точно (по сравнению с существующими аппроксимациями) описывает полевую зависимость критического тока ВТСП ҮВСО123 в широком диапазоне амплитуд магнитных полей, а в работе [13] показана адекватность данного приближения при описании ВТСП пленок разных производителей при различных температурах.

Связь магнитного поля $\mathbf{B}(X, Y, Z, t)$ с электрическим полем $\mathbf{E}(X, Y, Z, t)$ определяется законом индукции

$$
\frac{\partial \mathbf{B}(X, Y, Z, t)}{\partial t}=-\nabla \times \mathbf{E}(Z, Y, Z, t) .
$$

На двумерной пленке

$$
\frac{\partial B_{Z}(X, Y, 0, t)}{\partial t}=-\frac{\partial E_{X} X, Y, 0, t}{\partial Y}+\frac{\partial E_{Y} X, Y, 0, t}{\partial X} .
$$

Завершает систему уравнений нелинейный закон Ома для ВТСП пленки

$$
\mathbf{E}(X, Y, 0, t)=\rho(\mathbf{j}(X, Y, t), B(X, Y, 0, t)) \cdot \mathbf{j}(X, Y, t) .
$$

Зависимость двумерного удельного сопротивления $\rho(\mathbf{j}, B)$ часто выбирается в виде

$$
\begin{gathered}
\rho(\mathbf{j}, B)=\rho_{0} \cdot\left[|\mathbf{j}| / j_{c}(B)\right]^{n}, \\
\mathbf{j}=\sqrt{j_{X}^{2}(X, Y, t)+j_{Y}^{2}(X, Y, t)}
\end{gathered}
$$

(см., например, $[7,14])$, где обычно $n \sim 20-50$. В настоящей работе, также как в $[13,14]$, мы выбираем зависимость $\rho(\mathbf{j}, B)$ следующим образом:

$$
\rho(\mathbf{j}, B)= \begin{cases}0, & |\mathbf{j}|<j_{c}(B) \\ \rho_{0} \cdot\left[|\mathbf{j}|-j_{c}(B)\right]^{2}, & j_{c}(B) \leq|\mathbf{j}|\end{cases}
$$

Детальное описание алгоритма численного решения данной задачи представлено в работах $[13,15]$, и ввиду громоздкости, в данной статье не приводится. Представленная модель применялась нами в работах [13,15-17], где адекватно (соответствуя экспериментальным данным) описывала сверхпроводящие характеристики пластин ВТСП различной формы.

Уравнение Био-Савара дает взаимно-однозначное соответствие только между магнитным полем и двумерным током плоскости. Для восстановления плотности тока в сверхпроводящем слое конечной толщины (и определения зависимости $J_{c}\left(v(Z), B_{Z}\right)$ ) необходимо проводить магнитометрические исследования как на исходном образце, так и на образце с частично удаленным (истонченным) ВТСП-слоем. Предполагается, что контролируемое изменение толщины ВТСП-слоя проводится после однородного (в плоскости $(X, Y))$ облучения образца. В разделе 2.4 настоящей работы предлагается профиль толщины ВТСП-слоя, позволяющий проводить магнитометрические эксперименты для определения зависимости от координаты $Z$ токонесущих характеристик сверхпроводника. 

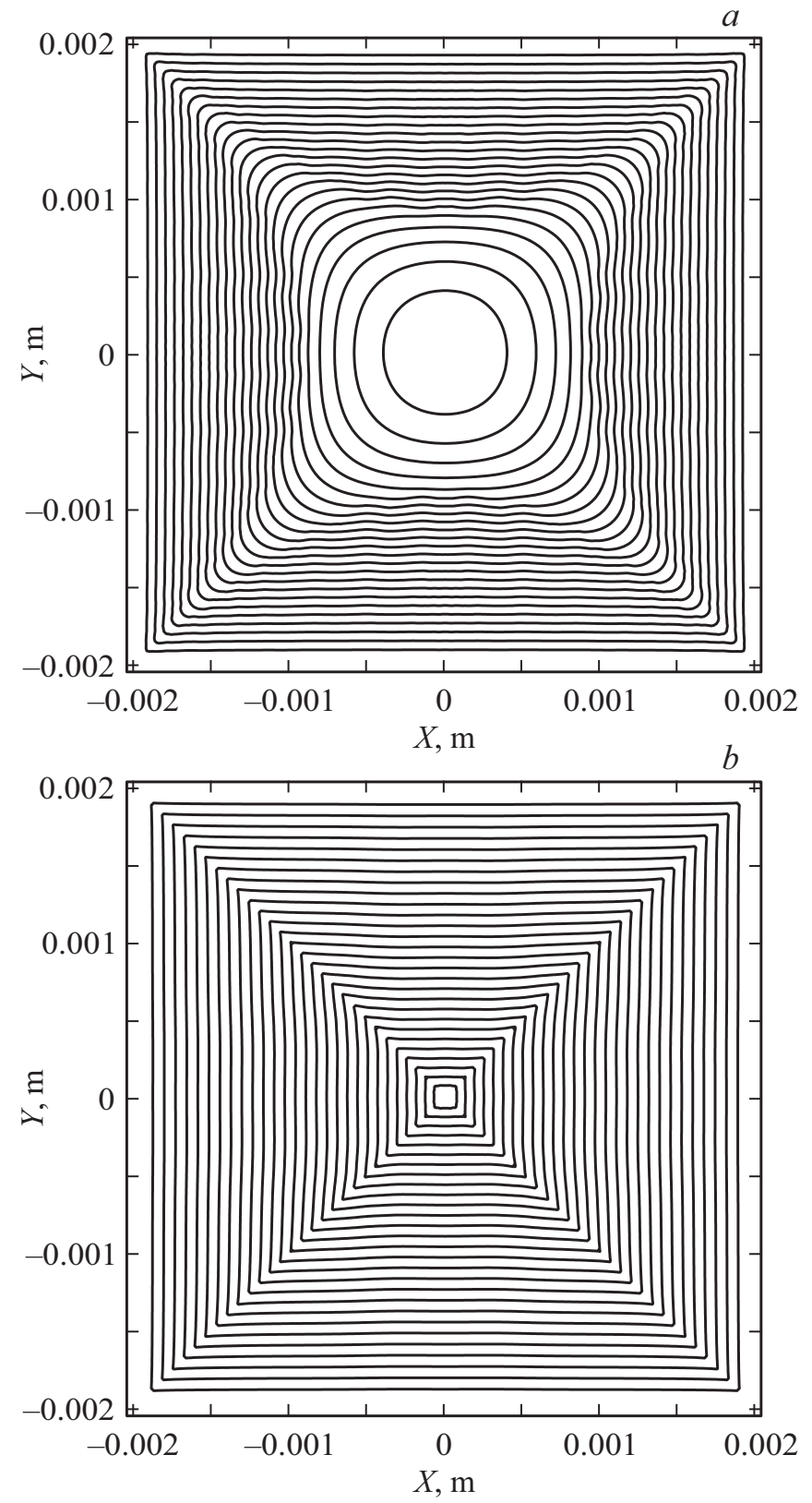

Рис. 4. Линии сверхпроводящих токов, наведенных внешним магнитным полем $B_{Z}$. Одна линия соответствует величине тока, равной $2 \mathrm{~A} . B_{Z}=0.01 \mathrm{~T}(a), B_{Z}=0.1 \mathrm{~T}(b)$.

\section{3. Выбор амплитуды магнитного поля и дозы облучения}

В соответствие с моделью критического состояния $[10,11]$ определить величину двумерного критического тока исходя из амплитуды тока наведенного можно только в случае, когда основная часть образца находится в критическом состоянии (см., например, [6,7]). Это определяет нижнюю границу амплитуды внешнего магнитного поля, индуцирующего сверхпроводящие токи. Еще одним фактором, ограничивающим снизу величину внешнего поля, является вопрос удобства. Наиболее точно численная модель описывает экспериментальную ситуацию в случае, когда внешнее магнитное поле преобладает над магнитным полем наведенных токов и нет необходимости учитывать наведенное магнитное поле в зависимости $j_{c}(B)[13]$. На рис. 4, $a$ представлены линии наведенных токов при слабом индуцирующем магнитном поле, когда плотность наведенного тока в центральной области образца ниже критической. Проведенные нами расчеты показывают, что этой области наблюдается полное экранирование внешнего магнитного поля. На рис. $4, b$ представлены линии тока при величине индукции внешнего магнитного поля $B_{Z}=0.1 \mathrm{~T}$, где область экранирования практически отсутствует, что характерно для критического состояния. На рис. 4, $b$ ясно видны токовые домены - области в которых линии токов не имеют изломов, а на границах наблюдается излом. Доменная структура является токов также является косвенным признаком критического состояния. Величина индукции внешнего магнитного поля $B_{Z}=0.1 \mathrm{~T}$ также является граничной, при которой влиянием собственного поля наведенных токов на величину $j_{c}(B)$ можно пренебречь. Поскольку в дальнейшем предполагается модельный расчет образцов, критический ток в которых ослаблен облучением, индукция внешнего магнитного поля $B_{Z}=0.1 \mathrm{~T}$ с запасом удовлетворяет требованиям, предъявляемым к нижней границе этой величины.

При проведении модельных расчетов мы выбираем дозу облучения, которая приводит к двукратному уменьшению плотности критического тока $j_{c}$. Выражения (2) и (3) позволяют определить зависимость объемной плотности критического тока от вертикальной координаты $Z$ после облучения следующим образом:

$$
J_{c}(Z)=\left.J_{c}\right|_{v=0} \cdot\left(1-0.5 \cdot h \cdot v_{\text {norm }}(Z)\right), \quad 0 \leq Z \leq h .
$$

Действительно, подстановка выражения (12) в формулу (1) дает значение критического тока $j_{c}$, в два раза меньшее этой величины в необлученном образце.

\section{4. Выбор формы маски и профиля толщины ВТСП-слоя}

Основное назначение маски в настоящей работе заключается в упрощении выбора дозы облучения, необходимой для двукратного подавления критического тока. Применение маски позволяет на одном образце сравнить критические токи поврежденной и неповрежденной части сверхпроводящей пленки. На рис. 5, a представлены линии токов в образце, верхняя часть которого $(Y>0)$ подверглась облучению, вследствие чего критический ток уменьшился в 2 раза. На этом рисунке видно, что густота токовых линий (определяющая плотность тока) в верхней части рисунка в два раза ниже аналогичной величины в неповрежденной области. Перспективной выглядит также маска в виде щели, состоящая из двух близко расположенных друг к другу экранов. Повреждаемая область в этом случае имеет вид узкой полосы, величина критического тока 
в которой также составляет половину исходного. Дополнительное преимущество такой маски в том, что она может применяться как в бесконтактной, так и в контактной методиках. Отметим, что применение маски возможно не только при радиационных воздействия, но и непосредственно при нанесении ВТСП-слоя, а также при ионной имплантации $[18,19]$. В контактной методике через образец пропускается транспортный ток, а в этом случае электрические контакты должны быть закрыты маской от облучения. На рис. 5, $b$ представлены линии токов в образце с облученной экваториальной полосой, ширина которой равна $0.4 \mathrm{~mm}$. На этом рисунке
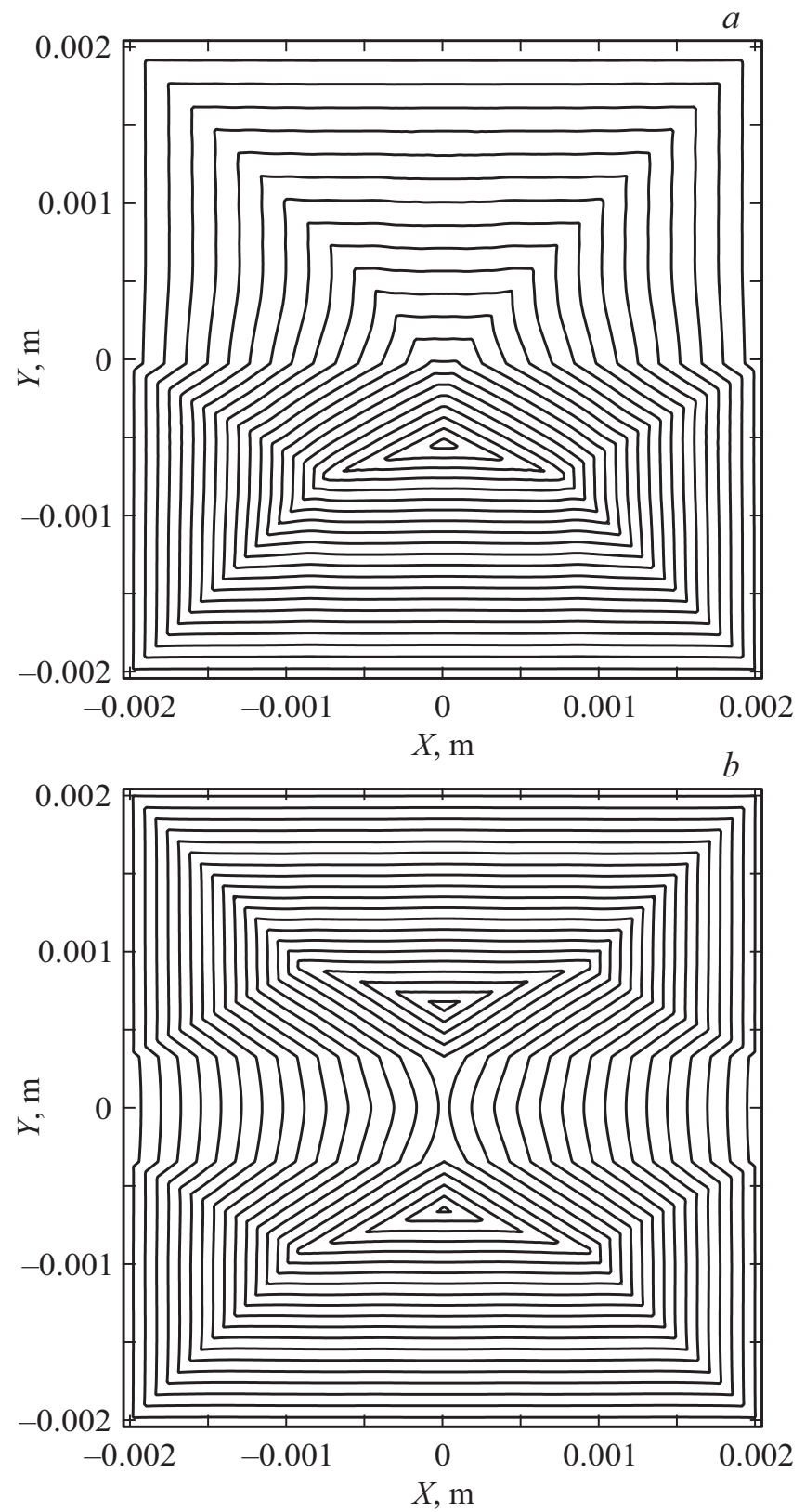

Рис. 5. Линии сверхпроводящих токов, наведенных внешним магнитным полем $B_{Z}$. Одна линия соответствует величине тока, равной $2 \mathrm{~A} . B_{Z}=0.1 \mathrm{~T}$. Образец с облученной верхней частью $(a)$ и с облученной центральной полосой $(b)$.

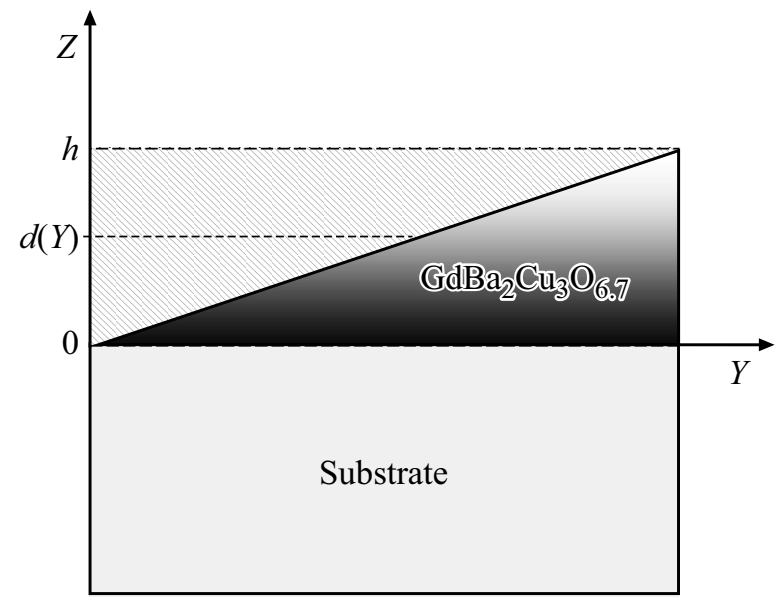

Рис. 6. Сечение сверхпроводящего клина. Неоднородность закраски клина иллюстрирует зависимость величины $J_{c}(Z)$. $d(Y)$ - толщина клина при соответствующем значении координаты $Y$.

также ясно видна полоса с пониженной в два раза плотностью тока.

При исследовании неоднородности критических характеристик ВТСП-слоя в вертикальном направлении применение маски не требуется. образец в плоскости $(X, Y)$ облучается однородно. Обратная задача Био-Савара позволяет определить величину двумерной плотности наведенного тока $j_{c}\left(B_{Z}\right)$. Определение трехмерной плотности критического тока $j 3 D_{c}\left(Z, B_{Z}\right)$ может быть осуществлено многократным однородным уменьшением толщины ВТСП-слоя с одновременным определением величины $j_{c}\left(B_{Z}\right)$ после каждого истончения. Такой подход требует трудоемкой серии измерений. Альтернативой может быть магнитометрическое измерение образца, сверхпроводящий слой которого имеет форму клина. Если после облучения будет проведен косой срез сверхпроводящего слоя (см. рис. 6), определение величины $j_{c}\left(B_{Z}\right)$ позволит восстановить искомую зависимость $J_{c}\left(Z, B_{Z}\right)$.

Обозначим переменной $j k_{c}(Y)$ величину двумерной плотности критического тока ВТСП-клина. Для облученного образца аналог выражения (7) для клина может быть представлен в следующем виде:

$$
\begin{gathered}
j k_{c}\left(Y, B_{Z}\right)=\int_{0}^{d(Y)} J_{c}\left(v(Z), B_{Z}\right) d Z, \\
d(Y)=h \cdot(Y+L / 2) / L .
\end{gathered}
$$

Из выражений (1), (12), (13) получаем следующее выражение

$$
j k_{c}\left(Y, B_{Z}\right)=\left.J_{c}\right|_{v=0} \cdot h \cdot\left[\frac{(Y+L / 2)}{L}-0.5 \cdot \int_{0}^{d(Y)} v_{n o r m}(Z) d Z\right] .
$$


Выражение (14) при решении прямой задачи (5-11) позволяет определить плотность наведенного магнитного момента и сверхпроводящие токи в ВТСП-клине с радиационными дефектами.

\section{3. Результаты и их обсуждение}

На рис. 7 представлены линии токов сверхпроводящего клина с однородным распределением дефектов. Линии токов в образцах с однородным и неоднородным (в вертикальном направлении) радиационным повреждением визуально мало различаются между собой. Для определения различий в образцах более удобна зависимость величины намагниченности $g(X=0, Y)$ от координаты $Y$ (на рис. 7 соответствующий отрезок сканирования отмечен пунктирной линией). На рис. 8 пунктирной жирной линией представлена эта зависимость для образца с однородным повреждением.

Для сильных магнитных полей $\left(\left(B_{\text {ext }}-B_{B} Z\right) /\right.$ $\left.B_{\text {ext }} \ll 1\right)$ функция $g(X=0, Y)$ на отрезке сканирования $X=0,-L / \leq Y \leq L / 2$ является гладкой за исключением единственной точки $Y=Y_{\min }$, в которой достигает своего минимального значения $\left(g\left(X=0, Y_{\min }\right)=g_{\min }\right.$, см. рис. 7,8$)$. Отсутствие изломов токовых линий позволяет установить для ВТСП-клина простую связь между величинами $j k_{c}(Y)$ и $g(X=0, Y)$. Вследствие симметрии токовых линий относительно отрезка сканирования компонента наведенного тока $j_{Y}=0$. В сильных магнитных полях основная часть сверхпроводящей пленки находится в критическом состоянии, для которого выполнено равенство что с учетом выражения (4) для отрезка сканирования дает следующее равенство $j_{c}(X, Y)=\sqrt{j_{X}^{2}(X, Y)+j_{Y}^{2}(X, Y)}$, что с учетом выражения (4) для отрезка сканирования дает следующее равенство

$$
j_{c}(X=0, Y)=\left|\frac{\partial g(X=0, Y)}{\partial Y}\right|=\left|j_{X}(X=0, Y)\right| .
$$

Из выражений (15) и (13) для ВТСП-клина получаем следующее уравнение:

$$
\begin{gathered}
\left|\frac{\partial g(X=0, Y)}{\partial Y}\right|=\int_{0}^{d(Y)} J_{c}\left(v(Z), B_{\text {ext }}\right) d Z, \\
d(Y)=h \cdot(Y+L / 2) / L,
\end{gathered}
$$

дифференцируя которое по переменной $Y$ получаем следующее выражение:

$$
\begin{gathered}
J_{c}\left(v(Z=d(y)), B_{\text {ext }}\right)=\frac{\partial}{\partial Y}\left(\left|\frac{\partial g(X=0, Y)}{\partial Y}\right|\right), \\
d(Y)=h \cdot(Y+L / 2) / L .
\end{gathered}
$$

При магнитометрическом восстановлении величины плотности компоненты магнитного момента пленки

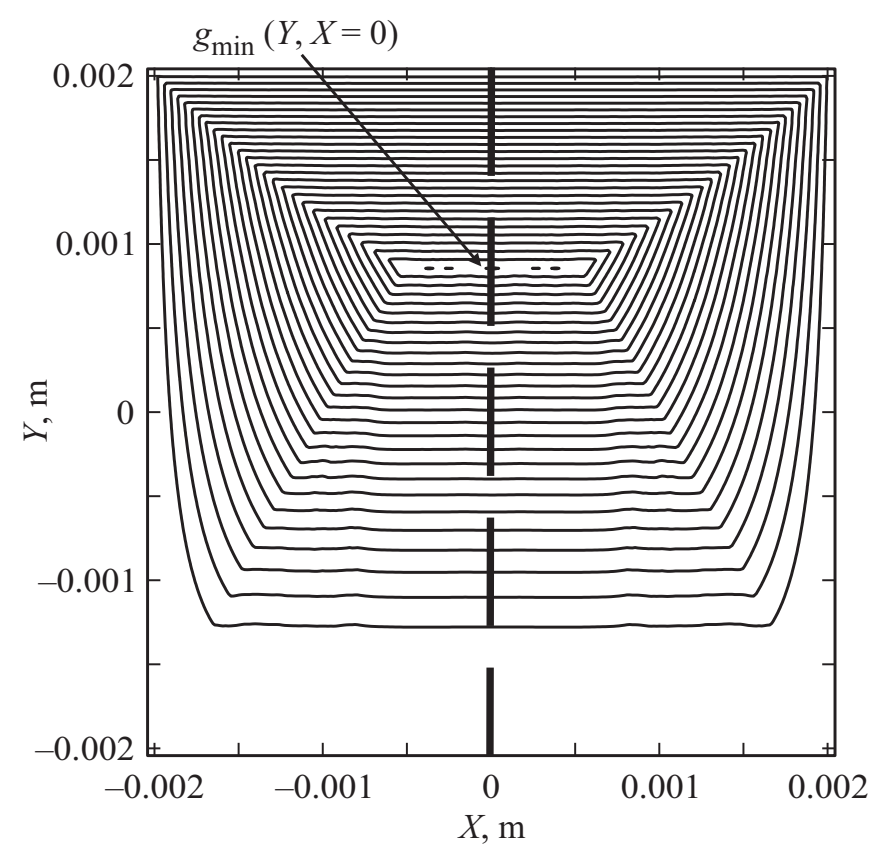

Рис. 7. Линии сверхпроводящих токов клина, наведенных внешним магнитным полем $B_{Z}$. Одна линия соответствует величине тока, равной 0.5 А. $B_{Z}=0.1 \mathrm{~T}$.

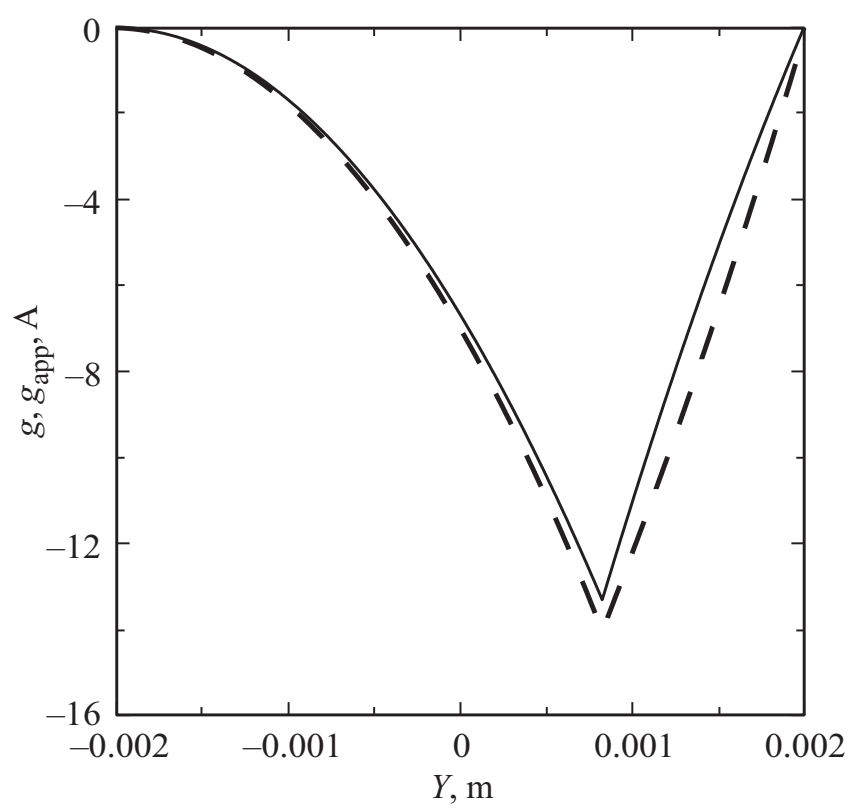

Рис. 8. Плотность распределения магнитного момента ВТСПпленки. Жирная черная пунктирная линия - результаты решения задачи (4-11) при гомогенном повреждении. Тонкая сплошная линия - аналитическое приближение (зависимость (18)).

$g(X, Y)$ выражение (17) однозначно определяет величину $J_{c}(Z)$. В приближении сильного магнитного поля $\left(\left(B_{e x t}-B_{Z}\right) / B_{e x t} \ll 1\right)$ при однородном повреждении сверхпроводника $J_{c}(Z)=\operatorname{const}(Z)=C\left(B_{\text {ext }}\right) / 2 h$, выражение (17) можно рассматривать как дифференциаль- 
ное уравнение относительно величины $g(X=0, Y)$ как непрерывной функции координаты $Y$. Учитывая выражение (6) и граничные условия $g(X=0, Y= \pm L / 2)=0$, приближенное значение плотности магнитного момента $g_{a p p}(Y, B)$ имеет следующий вид:

$$
\begin{gathered}
g_{a p p}\left(Y, B_{\text {ext }}\right)=C\left(B_{\text {ext }}\right) \cdot S(y+L / 2) / 4, \\
S(q)=\left\{\begin{array}{ll}
q^{2}, & 0 \leq q<L / \sqrt{2} \\
(L-q) \cdot\left[\frac{L}{\sqrt{2}-1}-q\right], & L \geq q \geq L \sqrt{2}
\end{array} .\right.
\end{gathered}
$$

Минимальное значение плотность магнитного момента, определяемого выражением (18) достигается в точке излома $Y_{\min }=L()$ и равно следующему значению:

$$
g_{\min }=-C \cdot\left(B_{e x t}\right) L / 2 .
$$

На рис. 8 представлена эта зависимость и решение задачи (4-9) для образца с однородным распределением дефектов при значении величины индукции внешнего магнитного поля $B_{e x t}=0.1 \mathrm{~T}$. Близкое расположение этих кривых на рис. 8 свидетельствует о слабом влиянии на плотность критического тока магнитного поля наведенных токов.

На рис. 9 и 10 представлена зависимость $g(X=0, Y)$ для образца с однородным повреждением, а также для образцов I, II и III, поврежденных ионами водорода и гелия, соответственно (см. таблицу). На этих рисунках заметно различное поведение неоднородно поврежденных образцов. Обращает на себя внимание почти идентичное поведение образцов I, II и III, вне зависимости от

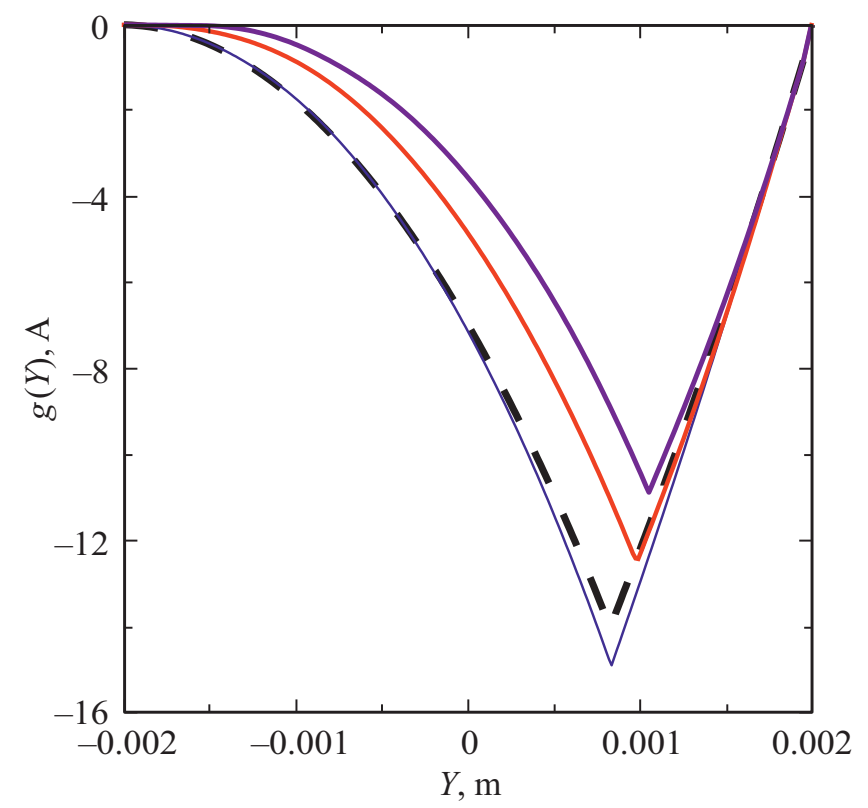

Рис. 9. Плотность распределения магнитного момента ВТСПпленки, облученной ионами водорода. Жирная черная пунктирная линия - гомогенное повреждение. Тонкая синяя линия образец I. Красная линия средней толщины - образец II. Жирная сплошная фиолетовая линия - образец III.

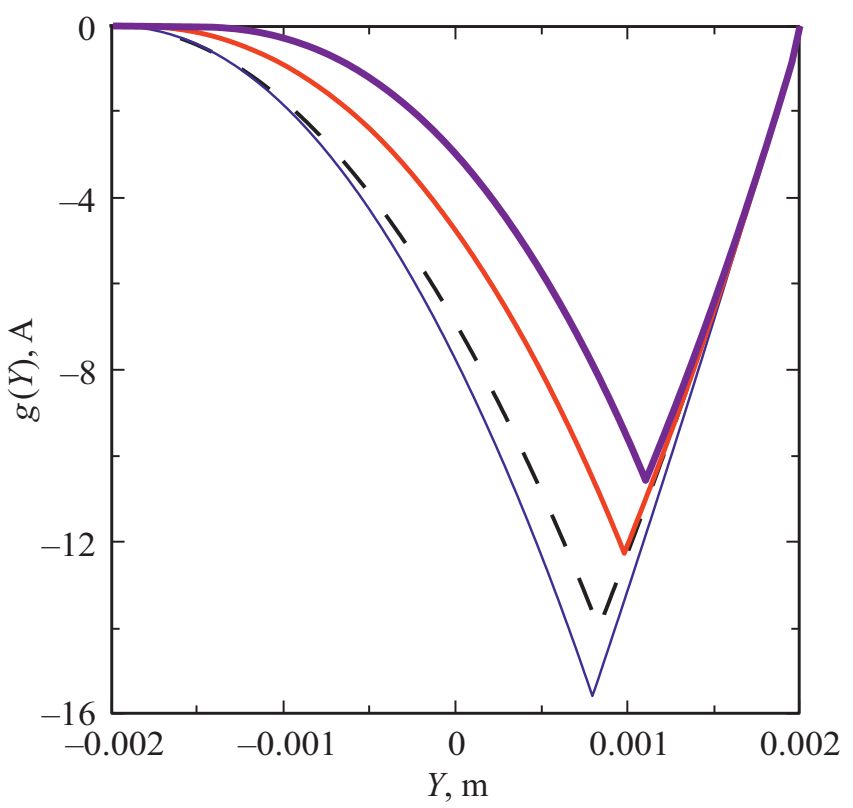

Рис. 10. То же, что на рис. 9 для пленки, облученной ионами гелия.

того какими ионами они облучались. Такое совпадение получено, несмотря на то что профили концентраций радиационных дефектов при облучении разными ионами (см. рис. 2, $a, b$ ) заметно различаются.

\section{4. Заключение}

Представленные в настоящей работе результаты моделирования позволяют сформулировать алгоритм экспрессной экспериментальной методики, позволяющей оценить неоднородность критического тока сверхпроводящего слоя, которая возникла в результате облучения сверхпроводника быстрыми заряженными частицами. Экспрессной эта методика может считаться, поскольку требует при реализации минимального количества технологических операций. Данная методика может применяться для исследования неоднородности сверхпроводящих свойств ВТСП-слоя во внешних магнитных полях. Показано, что для изучаемого образца сверхпроводящего слоя $\mathrm{GdBaCuO} 123$ методика может применяться при значении величины индукции внешнего магнитного поля $B_{\text {ext }}=0.1 \mathrm{~T}$ и выше.

Моделирование методики для ВТСП-композита было проведено при облучении ионами водорода и гелия. Энергии ионов были таковы, что быстрые частицы проходили через ВТСП-слой и останавливались в области подложки, граничной со сверхпроводником. Была обнаружена следующая особенность радиационной деградации сверхпроводящих свойств ВТСП-пленки. После облучения образцов сверхпроводящего композита с различным покрытием, неоднородность критического тока по толщине существенно зависела от характера покрытия, но не зависела от типа облучения. Учитывая 
универсальный (не зависящий от типа облучающих частиц) характер неоднородности, в дальнейшем было бы интересно расширить проведенные исследования, включив в список быстрых частиц ионы более тяжелых элементов.

Отметим, что аналогом радиационных воздействий может быть создание сквозных дефектов, например, с помощью лазерного воздействия. Как расчетные исследования [20,21], так и экспериментальные данные [22] указывают на возможность не только деградации свойств, но и увеличения критического тока. Вопрос контроля однородности ВТСП-пленки при таком воздействии по прежнему является актуальным.

\section{Финансирование работы}

Исследование выполнено при финансовой поддержке РФФИ и Госкорпорации „Росатом“ в рамках научного проекта № 20-21-00085.

\section{Конфликт интересов}

Авторы заявляют, что у них нет конфликта интересов.

\section{Список литературы}

[1] В.В. Деревянко, Т.В. Сухарева, В.А. Финкель. ФТТ 60, 465 (2018).

[2] P. Biersack, L.G. Haggmark. Nucl. Instrum. Meth. Phys. Res. B 74, 257 (1980). WWW.srim.org.

[3] Л.Х. Антонова, А.В. Троицкий, Г.Н. Михайлова, Т.Е. Демихов, С.В. Самойленков, А.А. Молодык, J. Noudem, P. Bernstein. Кр. сообщения по физике 44, 16 (2017). DOI: $10.3103 / \mathrm{S} 1068335617030034$

[4] П.И. Безотосный, С.Ю. Гаврилкин, К.А. Дмитриева, А.Н. Лыков, А.Ю. Цветков. ФТТ 61, 234 (2019).

[5] В.Ф. Елесин, И.А. Руднев. Сверхпроводимость: физика, химия, техника 4, 2055 (1991).

[6] Ch. Jooss, J. Albrecht, H. Kuhn, S. Leonhardt, H. Krounmuller. Rep. Prog. Phys. 65, 651 (2012).

[7] Th. Schuster, H. Kuhn, E.H. Brandt, M.V. Indenbom, M. Kläser, G. Müller-Vogt, H.U. Habermeier, H. Kronmüller, A. Forkl. Phys. Rev. B 52, 10375 (1995).

[8] A. Tikhanov. Sov. Math. Dokl. 4, 1035 (1963).

[9] D.M. Feldman. Phys. Rev. B 69, 144515 (2004).

[10] C.P. Bean. Rev. Mod. Phys. 36, 31 (1964).

[11] Y.B. Kim, C.F. Hempstead, A.R. Strand. Phys. Rev. Lett. 9, 306 (1962).

[12] A.I. Podlivaev, I.A. Rudnev, N.P. Shabanova. Bull.Lebedev Phys. Institute 41, 351 (2014).

[13] A.I. Podlivaev, I.F. Rudnev. Supercond. Sci. Technol. 30, 035021 (2017). doi.org/10.1088/1361-6668/aa55aa

[14] G. Iannone, S. Farinon, G. De Marzi, P. Fabricattore, U. Gambardella. IEEE Trans. Appl. Supercond. 25, 8200107 (2015).

[15] I.A. Rudnev, A,I. Podlivaev. IEEE Trans. Appl.Supercond. 26, 8200104 (2016). DOI: 10.1109/TASC.2016.2516347

[16] A.I. Podlivaev, I.V. Anischenko, S.V. Pokrovskii, I.A. Rudnev. IEEE Trans. Appl. Supercond. 28, 1 (2018).
[17] I.A. Rudnev, M. Osipov, S. Pokrovskii, A.I. Podlivaev. Mater. Res. Express Exp. 6, 036001 (2019). DOI: 10.1088/20531591/aaf7ae

[18] Д.В. Мастеров, С.А. Павлов, А.Е. Парафин, Е.В. Скороходов, П.А. Юдин. ФТТ 60, 2100 (2018).

[19] А.В. Антонов, А.В. Иконников, Д.В. Мастеров, А.Н. Михайлов, С.В. Морозов, Ю.Н. Ноздрин, С.А. Павлов, А.Е. Парафин, Д.И. Тетельбаум, С.С. Уставщиков, П.А. Юнин, Д.А. Савинов. ФТТ 61, 1573 (2019).

[20] А.Н. Максимова, В.А. Кашурников, А.Н. Мороз, И.А. Руднев. ФТТ 63, 65 (2021).

[21] A.N. Moroz, A.N. Maksimova, V.A. Kashurnikov, I.A. Rudnev. IEEE Trans. Appl. Supercond. 28, 8000705 (2018).

[22] S.V. Pokrovskii, O.B. Mavritskii, A.N. Egorov, N.A. Mineev, A.A. Timofeev, I.A. Rudnev. Supercond. Sci. Technol. 32, 075008 (2019).

Редактор К.В. Емцев 